\title{
A New Method for Solving a Class of Limit Problems in Statistical Analysis Teaching
}

\author{
Xiaonan Xiao \\ Xiamen University Tan Kah Kee College \\ Zhangzhou Fujian China \\ xiaoxn@xujc.com
}

\begin{abstract}
Statistical analysis is a branch of mathematics with rich content and wide application. Based on the theory of probability and mathematical statistics, it studies random phenomena by analyzing the data obtained from experiments or observations to achieve various reasonable estimations and inferences on the objective regularity of the research objects. Therefore, it is necessary to collect, organize and analyze the random data effectively, and to make as accurate and satisfactory an analysis as possible of the observed problems. In this way we build a necessary foundation for further solving practical problems.
\end{abstract}

Keywords-statistical analysis; teaching; limit problem; new method of solving

\section{INTRODUCTION}

In statistical analysis, using equivalent infinitesimal to find the limit can simplify the calculation. However, many people often use advanced infinitesimal because they do not consider advanced infinitesimal carefully. In this paper, on the basis of in-depth analysis of the causes of common errors in using equivalent infinitesimal to find the limit, a new method of using Taylor formula to replace the limit is proposed, which provides a new way to solve this kind of statistical analysis limit problem $^{[1-3]}$

\section{ANALYSIS OF COMMON ERRORS IN SOLVING LIMITS BY EQUIVALENT INFINITESIMAL SUBSTITUTION}

Generally speaking, Using Equivalent Infinitesimal Substitution to find the limit only works for multiplication or division of two infinitesimal quantities.

For the limit of two infinitesimal quantities which are non-multiplicative and non-divisive, we must give careful consideration to it ${ }^{[4]}$

For example when solving $\lim _{x \rightarrow 0} \frac{\operatorname{tg} x-\sin x}{x^{3}}$, the correct solution should be

$$
\begin{aligned}
& \lim _{x \rightarrow 0} \frac{\operatorname{tg} x-\sin x}{x^{3}}=\lim _{x \rightarrow 0} \frac{\sin x(1-\cos x)}{x^{3} \cos x}=\lim _{x \rightarrow 0} \frac{\sin x \cdot 2 \sin ^{2}\left(\frac{x}{2}\right)}{x^{3} \cos x}= \\
& \lim _{x \rightarrow 0}\left(\frac{\sin x}{x} \cdot \frac{\sin ^{2}\left(\frac{x}{2}\right)}{2 \cdot\left(\frac{x}{2}\right)^{2}} \cdot \frac{1}{\cos x}\right)=\frac{1}{2}
\end{aligned}
$$

But it is easy to make the following mistakes, that is when $x \rightarrow 0, \operatorname{tg} x \sim x, \sin x \sim x$, we can directly get $\lim _{x \rightarrow 0} \frac{\operatorname{tg} x-\sin x}{x^{3}}=$ $\lim _{x \rightarrow 0} \frac{x-x}{x^{3}}=\infty$. Obviously, the above limit is still the infinitive " $\frac{0}{0}$ ". Then the result is wrong. What is wrong with the above solution? It may be analyzed as follows. If the Taylor formula is used to expand the functions in the numerator at $\mathrm{X}=0$ until they have the same power as the denominator,

$$
\begin{aligned}
& \operatorname{tg} x=x+\frac{x^{3}}{3}+o\left(x^{3}\right) \\
& \sin x=x-\frac{x^{3}}{3}+o\left(x^{3}\right) \\
& \text { Then } \operatorname{tg} x-\sin x=\frac{x^{3}}{2}+o\left(x^{3}\right) \\
& \text { So } \lim _{x \rightarrow 0} \frac{\operatorname{tg} x-\sin x}{x^{3}}=\lim _{x \rightarrow 0} \frac{\frac{x^{3}}{2}+o\left(x^{3}\right)}{x^{3}}=\frac{1}{2}
\end{aligned}
$$

Thus, in the error solution, the principal part of two infinitesimal quantities is substituted for the difference formula, and the higher order infinitesimal of denominator is not omitted, so the result of calculation is wrong. It is not difficult to see from Formula (1) and Formula (2) that after $\operatorname{tg} \mathrm{x}$ and $\sin \mathrm{x}$ are expanded at $x=0$ to the same power as denominator. Later we pick $x+\frac{x^{3}}{3}$ to replace $\operatorname{tg} x$ and pick $x-\frac{x^{3}}{3}$ to replace $\sin x$. The higher order infinitesimal of denominator $x^{3}$ is omitted from the difference formula at this time. Then we get the correct result this time.

1) From all the above, we conclude that the solution limit can be obtained as $\lim _{x \rightarrow 0} \frac{f(x) \pm g(x)}{\rho(x) \pm \psi(x)}$. Thus a more effective Taylor Formula Substitution Method for $f(x) \pm g(x) \rightarrow 0$, $\rho(\mathrm{x}) \pm \psi(\mathrm{x}) \rightarrow 0, \mathrm{x} \rightarrow 0$ is :

2) The functions in denominator are expanded to n terms according to Taylor formula at $x=0$, and they are used to replace their respective functions. The result of merging the same items is taken as a new denominator. And $n$ is the minimum number of terms that make the new denominator not zero.

3) The functions in the molecule are expanded at $x=0$ until the terms with the same power as the new denominator are replaced by them, and the results of merging the same terms are taken as new molecules. 
4) Solve the limit of the fraction.

\section{A New MEthod of TAYLOR Formula REPLACEMENT FOR LIMIT}

Example 1: Seek the answer of $\lim _{x \rightarrow 0} \frac{\sin \alpha x-\sin \beta x}{x}$

Solution: Let us look at the denominator first. The expansion of $x$ is the linear term. Let us look at the numerator next. Expand $\sin \alpha x$ and $\sin \beta x$ to the first order Taylor Formula at $x=0$, then

$$
\begin{aligned}
& \sin \alpha x=\alpha x+o(\alpha x) \\
& \sin \beta x=\beta x+o(\beta x)
\end{aligned}
$$

Next, we use $\alpha x$ to replace $\sin \alpha x$ and use $\beta x$ to replace $\sin \beta x$. We get new numerator $\alpha x-\beta x=(\alpha-\beta) x$, So, the answer is $\lim _{x \rightarrow 0} \frac{(\alpha-\beta) \mathrm{x}}{\mathrm{x}}=\alpha-\beta$.

Example 2: Seek the answer of $\lim _{x \rightarrow 0} \frac{\rho^{x}-\sin x-1}{\ln (1+x)}$

Solution: Let us look at the denominator first. Just expand to the linear item $\ln (1+x)=x+0(x)$. The new denominator is $x$. Then let us look at the numerator. Because the new denominator is a linear term, the numerator functions only need to be expanded to linear term as

$$
e^{x}=1+x+o(x)
$$

$\sin x=x+o(x)$

Let us use $1+x$ to replace $e^{x}$ and use $x$ to replace $\sin x$. Then we get the new numerator as $(1+x)+x-1=2 x$. So, the answer is $\lim _{x \rightarrow 0} \frac{2 x}{x}=2$.

Example 3: Seek the answer of $\lim _{x \rightarrow 0} \frac{\cos x-e^{-\frac{x^{2}}{2}}}{x^{4}}$

Solution: Because the denominator is the $4^{\text {th }}$ order infinitesimal of $x$, it is only necessary to expand the functions in the numerator to include $x^{4}$, then we get

$$
\begin{aligned}
\cos x & =1-\frac{x^{2}}{2 !}+\frac{x^{4}}{4 !}+o\left(x^{4}\right) \\
e^{-\frac{x^{2}}{2}} & =1+\left(-\frac{x^{2}}{2}\right)+\frac{1}{2 !}\left(-\frac{x^{2}}{2}\right)^{2}+o\left[\left(-\frac{x^{2}}{2}\right)^{2}\right]=1-\frac{x^{2}}{2}+ \\
\frac{x^{4}}{8}+o\left(x^{4}\right) &
\end{aligned}
$$

And $\cos x-e^{-\frac{x^{2}}{2}}=-\frac{x^{4}}{12}+o\left(x^{4}\right)$

So, $\lim _{x \rightarrow 0} \frac{\cos x-e^{-\frac{x^{2}}{2}}}{x^{4}}=\lim _{x \rightarrow 0} \frac{-\frac{x^{4}}{12}+o\left(x^{4}\right)}{x^{4}}=-\frac{1}{12}$

If the above method is extended to the case of $\lim _{x \rightarrow 0} \frac{f(x) \pm g(x)}{\rho(x) \pm \psi(x)}, \quad(f(x) \pm g(x) \rightarrow 0, \rho(x) \pm \psi(x) \rightarrow 0)$ and the more general case of finding the algebraic sum of multiple functions, it is still correct ${ }^{[5-6]}$.
Example 4: Seek the answer of $\lim _{x \rightarrow 0}\left(x^{3}-x^{2}+\frac{x}{2} e^{\frac{1}{x}}-\right.$ $\left.\sqrt{1+x^{6}}\right)$

Analysis: This is an infinitive of $\infty$ - $\infty$ type. It can be replaced by using $t=\frac{1}{x}$ forming the infinitive formula $\frac{0}{0}$ of the upper type, and then expand $e^{t}$ and $\sqrt{1+t^{6}}$ according to the Taylor formula. We use the method similar to Example 3 to find the limit ${ }^{[7]}$.

Solution: Let $t=\frac{1}{x}$, then, when $x \rightarrow+\infty, t \rightarrow 0^{+}$.

Thus the original formula is equal to

$$
\begin{gathered}
\lim _{x \rightarrow 0}\left[\left(\frac{1}{t^{3}}-\frac{1}{t^{2}}+\frac{1}{2 t}\right) e^{t}-\right. \\
\left.\sqrt{1+\frac{1}{t^{6}}}\right]=\lim _{x \rightarrow 0} \frac{\left(1-t+\frac{t^{2}}{2}\right) e^{t}-\sqrt{1+t^{6}}}{t^{3}} \\
\text { and } e^{t}=1+t+\frac{t^{2}}{2}+\frac{t^{3}}{6}+o\left(t^{3}\right) \\
\sqrt{1+t^{6}}=1+\frac{t^{6}}{2}+o\left(t^{6}\right)
\end{gathered}
$$

So,

$$
\begin{aligned}
& \lim _{x \rightarrow 0}\left(x^{3}-x^{2}+\frac{x}{2} e^{\frac{1}{x}}-\sqrt{1+x^{6}}\right)= \\
& \lim _{\frac{1}{6}} \frac{\left(1-t+\frac{t^{2}}{2}\right)\left(1+t+\frac{t^{2}}{2}+\frac{t^{3}}{6}+o\left(t^{3}\right)\right)-\left(1+\frac{t^{6}}{2}+o\left(t^{6}\right)\right)}{t^{3}}=\lim _{x \rightarrow 0} \frac{\frac{t^{3}}{6}+o\left(t^{3}\right)}{t^{3}}=
\end{aligned}
$$

Let $\mathrm{f}(x)$ exist in the second derivative of $x=0$, and $\lim _{x \rightarrow 0}\left(\frac{\sin 3 x}{x^{3}}+\frac{f(x)}{x^{2}}\right)=0$, seek the answer of $f(0)$, $f^{\prime}(0), f^{\prime \prime}(0)$ and $\lim _{x \rightarrow 0}\left(\frac{3}{x^{2}}+\frac{f(x)}{x^{2}}\right)$

Analysis: Based on known conditions $\lim _{x \rightarrow 0}\left(\frac{\sin 3 x}{x^{3}}+\right.$ $\left.\frac{f(x)}{x^{2}}\right)=0$, if we expand $\sin 3 x$ and $f(x)$ according to the Taylor formula, we can analyze the satisfied condition of $f(0)$, $f^{\prime}(0), f^{\prime \prime}(0)$.

Solution: $\sin 3 x=3 x-\frac{1}{3 !}(3 x)^{3}+o\left(x^{3}\right)=3 x-\frac{9}{2} x^{3}+$ $o\left(x^{3}\right)$

$$
\begin{gathered}
f(x)=f(0)+f^{\prime}(0) x+\frac{1}{2} f^{\prime \prime}(0) x^{2}+o\left(x^{2}\right) \\
\frac{\sin 3 x}{x^{3}}+\frac{f(x)}{x^{2}} \\
=\frac{3 x-\frac{9}{2} x^{3}+f(0) x+f^{\prime}(0) x^{2}+\frac{1}{2} f^{\prime \prime}(0) x^{3}+o\left(x^{3}\right)}{x^{3}} \\
=\frac{(3+f(0)) x+f^{\prime}(0) x^{2}+\frac{1}{2}\left(f^{\prime \prime}(0)-9\right) x^{3}+o\left(x^{3}\right)}{x^{3}}
\end{gathered}
$$

Because $\lim _{x \rightarrow 0}\left(\frac{\sin 3 x}{x^{3}}+\frac{f(x)}{x^{2}}\right)=0$,

thus $3+\mathrm{f}(0)=0, f^{\prime}(0)=0, f^{\prime \prime}(0)-9=0$.

Then $\mathrm{f}(0)=-3, f^{\prime}(0)=0, f^{\prime \prime}(0)=9$ 


$$
\begin{aligned}
& \text { So, } \mathrm{f}(\mathrm{x})=-3+\frac{9}{2} x^{2}+o\left(x^{2}\right) \\
& \lim _{x \rightarrow 0}\left(\frac{3}{x^{2}}+\frac{f(x)}{x^{2}}\right)=\lim _{x \rightarrow 0} \frac{3-3+\frac{9}{2} x^{2}+o\left(x^{2}\right)}{x^{2}}=\frac{9}{2}
\end{aligned}
$$

\section{CONCLUSION}

Rogen Bacon, a great scientist, pointed out that "Mathematics is the key to open the door of science". c, this important key is also needed in statistical analysis ${ }^{[8-9]}$. With the advent of the global information age and the rapid development of modern science, technology and social economy, people will face more and more information decision-making and statistical analysis problems that must be solved in various fields. Information Decision-making and Statistical Analysis, as a new and practical comprehensive discipline, will provide more and more effective statistical decision-making analysis methods and means for the sustainable development of natural and Social Sciences in various fields in the process of its development to a higher level.

\section{REFERENCES}

[1] Bello Oyedele Adeshina, Bamiduro, Timothy Adebayo. Bootstrap Nonlinear Regression Application in a Design of an Experiment Data for Fewer Sample Size [J].International Journal of Research(Ijr),2015,2(2):1428-1441.

[2] Lorelied A Santos, Erniel B Barrios. Small Sample Estimation in Dynamic Panel Data Models: A Simulation Study[J].American Open Journal of Statistics,2011(1):58-73.

[3] Rui wang, Stephen w Lagakos, Robert j Gray. Testing and interval estimation for two-sample survival comparisons with small sample sizes and unequal censoring[J].Biostatistics, 2010, 11(4) :676-692.

[4] Harvey D I, Leybourne S J, Taylor A M R. Unit root testing in practice: dealing with uncertainty over the trend and initial condition[J].Econometric Theory,2009(25):587-636.

[5] Choi C, Moh Y. How useful are tests for unit-root in distinguishing unit-root process from stationary but non-linear process[J].Econometrics Journal,2007(10):82-112.

[6] Rodrigues P M. Properties of recursive trend-adjusted unit root tests[J].Economics Letters, 2006(91):413-419.

[7] Muller U K, Elliott G. Tests for unit roots and initial condition[J].Econometrica,2003(71): 1269-1286.

[8] Taylor R. Regression-based unit root tests with recursive mean adjustment for seasonal and non-seasonal time series[J].Journal of Business and Economic Statistics, 2002(20):269-281.

[9] Shin D W, So B S. Recursive mean adjustment for unit root tests[J].Journal of Time Series Analysis , 2001(22):595-612. 